\title{
Racial Disparities between the Sex Steroid Milieu and the Metabolic Risk Profile
}

\author{
Arlette Perry, ${ }^{1}$ Xuewen Wang, ${ }^{2}$ Ronald Goldberg, ${ }^{3}$ Robert Ross, ${ }^{4}$ and Loreto Jackson ${ }^{5}$ \\ ${ }^{1}$ Laboratory of Clinical and Applied Physiology, University of Miami, P.O. Box 248065, Coral Gables, FL 33124, USA \\ ${ }^{2}$ Division of Geriatrics and Nutritional Science, Washington University School of Medicine, 660 S. Euclid Avenue, \\ Louis street, MO 63110, USA \\ ${ }^{3}$ Diabetes Research Institute, Miller School of Medicine, University of Miami, 1450 NW 10th Avenue, Miami, FL 33136, USA \\ ${ }^{4}$ Department of Medicine, School of Kinesiology and Health Studies, Queen's University, Kingston, ON, Canada K7L 3N6 \\ ${ }^{5}$ Clemson University, Clemson, SC 29634, USA
}

Correspondence should be addressed to Arlette Perry, aperry@miami.edu

Received 30 September 2009; Revised 1 February 2010; Accepted 23 April 2010

Academic Editor: Eliot Brinton

Copyright (๑) 2010 Arlette Perry et al. This is an open access article distributed under the Creative Commons Attribution License, which permits unrestricted use, distribution, and reproduction in any medium, provided the original work is properly cited.

\begin{abstract}
Aims and Method. The present study examined the relationship between the metabolic risk profile (MRP) and total testosterone (TT) and free testosterone using the free androgen index (FAI) and sex hormone binding globulin (SHBG) in 36 Caucasian American (CA) and 30 African-American (AA) women volunteering for a weight loss study. Results. After controlling for age, significant relationships were found between TT and diastolic blood pressure $(P=.004$ and $P=.015$ in CA and AA women, resp.). Additionally, total cholesterol $(P=.003)$, low density lipoprotein cholesterol $(P=.004)$, apolipoprotein $\mathrm{B}(P=.006)$, and the total cholesterol/high density lipoprotein cholesterol $(P=.027)$ were significantly related to TT in AA women only. In CA women, similar measures of glucose/insulin status related to FAI, were also related to SHBG. In both CA and AA women, SHBG was related to waist $(P=.031$ and $P=.022$ resp.). Conclusion. Our findings showed racial disparities in the relationship between the sex steroid milieu and the MRP in overweight/obese CA and AA women.
\end{abstract}

\section{Introduction}

In women, a more android fat distribution pattern signified by increased visceral adipose tissue (VAT) is associated with high testosterone and low-sex hormone binding globulin (SHBG), adverse lipids and lipoproteins, and altered glucose/insulin homeostasis [1,2]. Since sex steroids and SHBG serve as a marker of androgen status, they may be considered a valuable marker of metabolic variables associated with cardiovascular disease (CVD) risk [3].

In a large study of both pre- and postmenopausal women, Bell and colleagues [4] confirmed the importance of SHBG as an independent predictor of the metabolic risk profile (MRP) in women across the lifespan. However, despite higher rates of CVD [5], obesity [6], and type II diabetes [7], few studies have targeted overweight or obese African-American (AA) women.

In premenopausal women in the SWAN study [8], investigators did compare racial groups and found a sig- nificant correlation between SHBG, testosterone, and the MRP in minority women. Their results showed consistent hormone-risk factor relationships across racial groups that in some instances appeared stronger in Asian and Caucasian women. They also showed significant differences between testosterone and triglycerides (TG) in Black and Hispanic women. However, due to large BMI variances found among racial groups, investigators cautioned against comparing relationships across racial lines. In contrast, Berman and colleagues [9] found several significant relationships between SHBG and the MRP in postmenopausal Caucasian American (CA) women despite the fact that no significant relationships were evidenced in postmenopausal AA women. Unfortunately, investigators failed to control for intra-abdominal or VAT in either study, leaving potential mediating processes uninvestigated across racial groups.

In a study of premenopausal Hispanic and CA women by Haffner and colleagues $[10,11]$ a significant inverse relationship between SHBG, insulin resistance, and atherogenic 
lipids was found to be similar across Hispanic and CA women supporting similarity in these relationships across racial lines. The similar and significant relationships observed in this study remained after controlling for BMI and VAT [10]. However, controlling for insulin negated a number of relationships that were previously significant, demonstrating the importance of insulin in modulating this relationship. In contrast, other investigations found that after controlling for VAT, neither SHBG nor testosterone were related to the MRP reaffirming the importance of VAT in modulating this relationship $[1,2]$.

Given the inconsistencies in the literature and lack of emphasis in overweight/obese women, the present study examined total testosterone (TT), free testosterone, using the free androgen index (FAI) and SHBG, in relation to MRP in a group of overweight/obese premenopausal CA and AA women. A secondary purpose was to examine whether these relationships were mediated by VAT or insulin in each group of women.

\section{Subjects and Methods}

2.1. Subjects. Participants were volunteers interested in a weight loss program. Subjects were required to be premenopausal, weight stable for a minimum of three months, possessing a BMI above 25 , and free of known metabolic disease such as diabetes, hyperlipidemia, hypertension, polycystic ovary syndrome, or CVD. Subjects who were pregnant or lactating, amenorrheic, or taking medications or hormones that would affect blood pressure (BP), carbohydrate, or lipid metabolism were excluded from the study. All subjects were required to be eumenorrheic having a normal monthly menstrual cycle for the proceeding six months. A total of 66 subjects (36 CA; $30 \mathrm{AA}$ ) met the criteria necessary for participation in the study. One weekday and one weekend day were randomly selected for testing and evaluation of participants. A second consecutive weekday and weekend day were also provided to maximize participation of all subjects. Subjects gave informed consent and completed all testing procedures in accordance with the ethical standards and guidelines of the Institutional Review Board guidelines for human subjects at the University of Miami.

2.2. Physical and Anthropometric Measurements. Body weight was measured to the nearest $0.1 \mathrm{~kg}$. Height was measured to the nearest $0.5 \mathrm{~cm}$, and BMI was calculated as weight $(\mathrm{kg})$ divided by the square of height $\left(\mathrm{m}^{2}\right)$. Waist circumference was measured using a spring tension measuring tape (Gays Mills, WI) midway between the lower rib margin and iliac crest, [12]. All anthropometric measurements were performed by the same investigator who recorded the mean of the two measurements to the nearest $1.0 \mathrm{~mm}$.

Following an overnight fast, systolic and diastolic blood pressure (SBP and DBP, resp.) were taken in one setting following a 5-min rest interval. Duplicate blood pressure measurements were taken from the left upper arm, averaged, and recorded to the nearest $2.0 \mathrm{mmHg}$.
2.3. Magnetic Resonance Imaging (MRI). The abdominal region was measured by MRI using a $1.5 \mathrm{~T}$ instrument (Siemens Medical Systems, Iselin, NJ). Spin-echo imaging was performed using a T1-weighted sequence with a 147$\mathrm{ms}$ repetition time and $4.8 \mathrm{~ms}$ echo time. Ten mm thick images with a $2.5-\mathrm{mm}$ gap between images were obtained during a single breath hold sequence. A total of seven images were obtained in each subject with the central slice obtained at the L4-L5 intervertebral disk space. VAT was defined as adipose tissue contained within the boundaries of the rectus abdominis, internal obliques, quadratus lumborum, and long back muscles while subcutaneous adipose tissue (SAT) was adipose tissue located between the skin and the same group of muscles. Both VAT and SAT were computed by summing the respective VAT and SAT partial volumes $\left(\mathrm{cm}^{3}\right)$ and converting to liters.

2.4. Serum Measurements. Blood was withdrawn from the antecubital vein after a 12 -h fast and following centrifugation. Serum was analyzed within one week of withdrawal. All serum measurements were taken while subjects were seated quietly for at least five minutes. Total cholesterol [13], highdensity lipoprotein cholesterol (HDL) [14], its subfractions [15], and TG [16] were measured by the Diabetes Research Institute Lipid Laboratory, University of Miami. Serum standards used for calibration were developed by the Diabetes Research Institute and calibrated against serum samples from the Centers for Disease Control and Prevention Laboratory, Atlanta, GA. Very low density lipoprotein cholesterol was estimated by TG divided by five, and low density lipoprotein cholesterol (LDL) was indirectly calculated by subtracting HDL and very low density lipoprotein cholesterol from total cholesterol [17].

The peak particle diameter for major LDL subfractions was determined on a gel scan based upon standards of known diameter as described by Krauss and Burke [18]. The LDL size of the predominant peak for a subject was identified as the subjects' LDL peak particle size (pps).

Apolipoprotein B in serum was measured by turbidimetric immunoassay using a commercially available kit (Incstar, Stillwater, MN) according to procedures outlined by the manufacturers. All apolipoprotein B procedures have been developed in accordance with the guidelines set forth by the International Federation of clinical chemistry.

Fasting glucose levels were determined spectrophotometrically at a wavelength of $340 \mathrm{~nm}$ using a hexokinase reaction developed by Roche (Roche Diagnostic System, Nutley, NJ). Serum insulin was measured by radioimmunoassay of serum using a Coat-A-Count insulin procedure (Diagnostic Products, Los Angeles, CA). The insulin resistance was assessed by using the homeostasis model assessment (HOMA) index, which divides the product of fasting insulin $(\mu \mathrm{U} / \mathrm{mL})$ and glucose ( $\mathrm{mmol} / \mathrm{L})$ by 22.5 [19].

Serum SHBG was analyzed by immunoradiometric assay (Siemens Health Care Diagnostics; Los Angeles, CA). The TT was analyzed by Coat-A-Count radioimmunoassay using a testosterone-specific antibody (Siemens Health Care Diagnostics; Los Angeles, CA). Quality control procedures for 
accuracy and reliability in the laboratory yielded intra-assay and interassay coefficients of variation of less than $5 \%$ for SHBG and less than $7.5 \%$ for TT. The FAI was the ratio of TT/SHBG in the same unit $[20,21]$.

2.5. Additional Assessments. Nutrient intake and alcohol consumption (g/day) were recorded using a three-day food log and analyzed using the Dine System (Buffalo, N.Y. 1994). Cigarettes smoked were determined using a medical history questionnaire. Subjects were administered the College Alumnus Questionnaire developed by Paffenbarger et al. [22] for evaluating daily physical activity. Education experience was measured using a 1-5 categorical scale modified from Rosmond et al. [23]. All subjects completed the Daily Stress Inventory which has been shown to be a significant correlate of endocrine measures of stress [24].

2.6. Statistical Analysis. All statistical analyses were completed using the Statistical Package for the Social Sciences, version 10.1 [25]. Means \pm standard deviations of all variables were calculated for participants. Natural log transformation was used for TG, SHBG, and FAI while the square root of TT was used to achieve normality of distribution. Student's $t$-tests for unpaired samples were used to assess differences between racial groups. Since age showed a trend toward significant differences in CA and AA women, Pearson's correlation coefficients were performed after controlling for age, to examine the relationship between SHBG and sex steroids with the MRP in CA and AA women. These correlations were also conducted after controlling for VAT and insulin.

\section{Results}

3.1. Descriptives. A total of 66 subjects, $36 \mathrm{CA}$ and $30 \mathrm{AA}$ women, were evaluated for subject characteristics. A comparison between groups indicated that CA women had significantly higher VAT and TG levels than AA women $(P<.05$ for both $)$. There was also a trend toward significant differences in waist circumference $(P=.058)$, age $(P=.063)$, and SHBG $(P=.073)$. No other significant differences or trends in metabolic variables were noted between groups.

\subsection{Association between Total Testosterone and the Metabolic} Risk Profile. Pearson's correlation coefficient showed a significant inverse correlation between TT and BP $(P=.014$ and $P=.004$ for SBP and DBP, resp.) indicating that higher TT was associated with lower BP in CA women. This was observed concomitant with a positive relationship between SAT and TT $(P=.006)$. In AA women, significant inverse relationships were also observed between TT and DBP $(P=$ $.015)$ as well as total cholesterol $(P=.003), \operatorname{LDL}(P=.004)$, Apo B $(P=.006)$, and total cholesterol/HDL $(P=.027)$. In both $\mathrm{CA}$ and $\mathrm{AA}$ women, all significant relationships remained, after controlling for VAT or insulin.

3.3. Association between Free Androgen Index and the Metabolic Risk Profile. Pearson correlation coefficient showed significant positive relationships between FAI and waist circumference $(P=.048)$, SAT $(P=.026)$, glucose, insulin, and HOMA IR $(P \leq .01$ for all $)$ and a negative relationship with $\operatorname{HDL}(P=.043)$ in CA women. Similar to what was observed for TT, significant relationships between FAI and DBP $(P=.018)$, total cholesterol $(P=.009)$, LDL $(P=.010)$, and Apo B $(P=.029)$ were found for AA women. Controlling for VAT had no impact upon aforementioned relationships in either CA or AA women. Controlling for insulin, however, negated all significant relationships between FAI and HOMA IR as well as serum lipid and lipoproteins in both CA and AA women.

3.4. Association between Sex Hormone Binding Globulin and the Metabolic Risk Profile. In CA women, SHBG was significantly and positively related to waist circumference $(P=.031)$, VAT $(P=.004)$, glucose $(P=.009)$, insulin $(P=.001)$, and HOMA IR $(P=.001)$. In AA women, the only significant associations were observed between SHBG and waist circumference $(P=.022)$. However, there was a trend toward significant relationships between SHBG and VAT $(P=.096)$, insulin $(P=.056)$, HOMA IR $(P=$ $.090)$, and $\operatorname{HDL}(P=.094)$ in AA women. Adjusting for insulin negated all significant correlations in both CA and AA women. Adjusting for VAT negated the significant relationship between SHBG and waist circumference in AA women.

\section{Discussion}

The present study revealed several interesting findings relevant to sex steroids, SHBG, and the MRP in CA and AA women. As expected, CA women showed higher VAT and TG levels than AA women, which had been reported in previous studies [26, 27]. After controlling for VAT, however, there were no longer significant differences in TG levels between groups indicating that lower TG in AA women were accounted for, in part, by their lower VAT levels. Controlling for VAT also eliminated any trends toward significance by group for waist circumference and SHBG, highlighting the importance of VAT in these relationships. In contrast, other studies have shown FAI to be significantly lower [8] and SHBG significantly higher [28] in Black compared to White subjects.

Interestingly, our findings showed an inverse relationship between TT and several metabolic variables observed in both CA and AA women (Table 2). The majority of research has suggested that women possessing higher androgens tend to display a more adverse MRP. Other studies, however, have shown no association between TT and the MRP $[1,2]$. Still others have reported favorable associations between TT and metabolic variables $[29,30]$. Equivocal results may be found because TT represents a combination of the more bioactive, free form as well as the inactive form bound with other proteins, that is, SHBG, albumin. In the present study, as TT went up, BP went down and this relationship was observed in both $\mathrm{CA}$ and $\mathrm{AA}$ women. 
TABLE 1: Comparison of subject characteristics between CA and AA women using student's $t$-tests for unpaired samples.

\begin{tabular}{|c|c|c|c|}
\hline Characteristics & CA women $(n=36)$ & AA women $(n=30)$ & $P^{\mathrm{a}}$ \\
\hline Age (y) & $41.94 \pm 7.10$ & $37.97 \pm 9.94$ & .063 \\
\hline Height (m) & $1.64 \pm 0.07$ & $1.62 \pm 0.06$ & .168 \\
\hline Weight (kg) & $94.79 \pm 15.72$ & $91.84 \pm 14.03$ & .427 \\
\hline $\operatorname{BMI}\left(\mathrm{kg} / \mathrm{m}^{2}\right)$ & $35.12 \pm 5.35$ & $34.88 \pm 4.4$ & .850 \\
\hline Resting heart rate (beats/min) & $73.67 \pm 11.04$ & $76.57 \pm 10.90$ & .289 \\
\hline Stress $^{\mathrm{b}}$ & $67.71 \pm 21.37$ & $69.28 \pm 31.64$ & .815 \\
\hline Education $^{\mathrm{c}}$ & $2.56 \pm 0.50$ & $2.30 \pm 0.66$ & .077 \\
\hline Physical activity (kcal/wk) & $983.65 \pm 1,033.60$ & $892.88 \pm 895.74$ & .723 \\
\hline Cigarettes (no./d) & $2.11 \pm 6.96$ & $1.53 \pm 5.10$ & .707 \\
\hline Alcohol & $5.56 \pm 13.78$ & $2.27 \pm 5.65$ & .226 \\
\hline \multicolumn{4}{|l|}{ Metabolic risk profile } \\
\hline Waist (cm) & $107.29 \pm 14.50$ & $101.03 \pm 11.27$ & .058 \\
\hline WHR & $0.86 \pm 0.06$ & $0.87 \pm 0.08$ & .536 \\
\hline SAT (L) & $4.68 \pm 1.28$ & $4.51 \pm 1.40$ & .603 \\
\hline VAT $(\mathrm{L})$ & $1.20 \pm 0.48$ & $0.76 \pm 0.30$ & $<.001$ \\
\hline Glucose $(\mathrm{mmol} / \mathrm{L})^{\mathrm{d}}$ & $4.80 \pm 0.57$ & $4.76 \pm 5.77$ & .758 \\
\hline Insulin $(\mu \mathrm{U} / \mathrm{mL})$ & $14.84 \pm 8.13$ & $14.75 \pm 5.77$ & .957 \\
\hline HOMA IR & $3.13 \pm 1.81$ & $3.20 \pm 1.39$ & .878 \\
\hline Systolic blood pressure (mmHg) & $126.67 \pm 14.89$ & $123.00 \pm 14.21$ & .313 \\
\hline Diastolic blood pressure $(\mathrm{mmHg})$ & $82.11 \pm 10.13$ & $82.20 \pm 8.70$ & .970 \\
\hline Triglycerides (mg/dL) & $144.50 \pm 78.44$ & $107.77 \pm 67.58$ & .048 \\
\hline Total cholesterol (mmol/L) & $5.34 \pm 1.32$ & $5.14 \pm 1.41$ & .552 \\
\hline $\mathrm{HDL}(\mathrm{mmol} / \mathrm{L})$ & $1.44 \pm 0.42$ & $1.45 \pm 0.29$ & .931 \\
\hline $\mathrm{HDL}_{2}(\mathrm{mmol} / \mathrm{L})$ & $0.36 \pm 0.23$ & $0.41 \pm 0.28$ & $.428^{\mathrm{e}}$ \\
\hline $\mathrm{LDL}(\mathrm{mmol} / \mathrm{L})$ & $3.10 \pm 0.96$ & $3.13 \pm 1.25$ & .909 \\
\hline LDL pps (nmol/L) & $27.11 \pm 0.71$ & $27.15 \pm 0.57$ & .799 \\
\hline Apo B (mg/dL) & $106.50 \pm 29.22$ & $102.70 \pm 30.69$ & .609 \\
\hline Total cholesterol/HDL & $3.91 \pm 1.26$ & $3.69 \pm 1.35$ & .488 \\
\hline $\mathrm{Lp}(\mathrm{a})(\mathrm{mg} / \mathrm{dL})$ & $24.86 \pm 20.60$ & $36.85 \pm 23.59$ & .093 \\
\hline \multicolumn{4}{|l|}{ SHBG and Sex Steroids } \\
\hline SHBG $(\mathrm{nmol} / \mathrm{L})^{\mathrm{e}}$ & $33.88 \pm 22.98$ & $43.80 \pm 30.73$ & $.073^{\mathrm{e}}$ \\
\hline $\mathrm{TT}(\mathrm{ng} / \mathrm{mL})^{\mathrm{e}}$ & $0.27 \pm 0.11$ & $0.33 \pm 0.19$ & $.170^{\mathrm{e}}$ \\
\hline FAI & $4.24 \pm 3.55$ & $4.11 \pm 4.83$ & $.685^{\mathrm{e}}$ \\
\hline
\end{tabular}

Values are means \pm SD. SAT indicates volume of subcutaneous adipose tissue; VAT indicates volume of visceral adipose tissue; IR, insulin resistance calculated as (insulin, $\mu \mathrm{U} / \mathrm{mL} \times$ glucose, $\mathrm{mmol} / \mathrm{L}$ ) $\div 22.5$; HDL, high-density lipoprotein cholesterol; LDL, low-density lipoprotein cholesterol; LDL pps-low-density lipoprotein, peak particle size; Apo B, apolipoprotein B; SHBG, sex hormone binding globulin; TT, total testosterone, FAI, free androgen index, calculated as $\mathrm{TT}(\mathrm{ng} / \mathrm{mL}) \times 3.467 \times 100 / \mathrm{SHBG}(\mathrm{nmol} / \mathrm{L})$.

avalues obtained from a Student's $t$-test for unpaired samples.

${ }^{\mathrm{b}}$ Graded numerically, with the higher number indicating a higher stress level.

${ }^{\mathrm{c}}$ Graded on a scale of 1 to 5 , with the higher number indicating a higher level of education.

${ }^{\mathrm{d}}$ One outlier was excluded from the analysis.

${ }^{\mathrm{e}} t$-test performed on transformed values.

Paradoxically TT has been reported to have both vasodilatory and vasoconstrictive effects [31]. According to Livingstone and Collison [32], there may be a "physiological window" within which normal androgen/estrogen levels confer health benefits and beyond which may show detrimental effects. Based upon the literature, our women demonstrated low normal levels of TT. If estrogen levels fell along the same lines, a more balanced androgen/estrogen ratio would have translated into a more favorable association between TT and the MRP.
Unexpected was the significant inverse relationship between TT and several lipoproteins in AA women. Total cholesterol/HDL is considered one of the most powerful predictors of CVD [33] and in AA women, Apo B, another marker of atherogenic risk, shared almost $28 \%$ of its variance with TT. Thus, at a given level of TT, AA women demonstrated lower levels of total cholesterol, LDL, and ApoB than CA women. This contrasts with other studies using minority groups $[8,34]$ which showed very little if any association between TT and the MRP. In the Swan study [8], TT was 
TABLE 2: Pearson's correlation coefficients between total testosterone (square root) and the metabolic risk profile after adjusting for age.

\begin{tabular}{|c|c|c|c|c|}
\hline Metabolic risk profile & $\begin{array}{c}\text { Caucasian American } \\
\text { women ( } r \text { value })\end{array}$ & $P$ value & $\begin{array}{l}\text { African American } \\
\text { women ( } r \text { value })\end{array}$ & $P$ value \\
\hline BMI $\left(\mathrm{kg} / \mathrm{m}^{2}\right)$ & 0.124 & .478 & -0.072 & .711 \\
\hline WAIST (cm) & 0.235 & .174 & -0.117 & .547 \\
\hline SAT (L) & 0.452 & .006 & -0.104 & .593 \\
\hline VAT (L) & 0.100 & .567 & 0.176 & .361 \\
\hline Glucose (mmol/L) & 0.220 & .211 & -0.02 & .924 \\
\hline Insulin $(\mathrm{u} \mu / \mathrm{mL})$ & 0.281 & .108 & 0.004 & .986 \\
\hline HOMA IR & 0.246 & .161 & 0.008 & .967 \\
\hline SBP (mmHg) & -0.411 & .014 & -0.298 & .116 \\
\hline DBP (mmHg) & -0.481 & .004 & -0.448 & .015 \\
\hline $\mathrm{TG}(\mathrm{mg} / \mathrm{dL})$ & 0.015 & .934 & -0.175 & .394 \\
\hline $\begin{array}{l}\text { Total cholesterol } \\
(\mathrm{mmol} / \mathrm{L})\end{array}$ & -0.259 & .140 & -0.555 & .003 \\
\hline $\mathrm{HDL}(\mathrm{mmol} / \mathrm{L})$ & -0.242 & .168 & -0.058 & .778 \\
\hline $\mathrm{HDL}_{2}(\mathrm{mmol} / \mathrm{L})$ & -0.025 & .888 & -0.267 & .187 \\
\hline $\mathrm{LDL}(\mathrm{mmol} / \mathrm{L})$ & -0.320 & .065 & -0.550 & .004 \\
\hline LDL pps (nmol/L) & -0.029 & .871 & 0.238 & .241 \\
\hline Apo B (mg/dL) & -0.239 & .173 & -0.528 & .006 \\
\hline $\begin{array}{l}\text { Total } \\
\text { cholesterol/HDL-C }\end{array}$ & -0.101 & .571 & -0.433 & .027 \\
\hline $\operatorname{Lp}(\mathrm{a})$ & -0.082 & .654 & -0.008 & .967 \\
\hline
\end{tabular}

SBP, systolic blood pressure, DBP, diastolic blood pressure, SAT, subcutaneous adipose tissue volume, VAT, visceral adipose tissue volume, HOMA IR, insulin resistance calculated as (insulin $\times$ glucose) $\div 22.5$, HDL, high density lipoprotein cholesterol, $\mathrm{HDL}_{2}$, high-density lipoprotein subfraction 2 , LDL, low-density lipoprotein cholesterol, Apo B, apolipoprotein B, LDL pps, low density lipoprotein peak particle size, TG, triglycerides, log transformed, BMI, body mass index, WAIST, waist circumference.

correlated with TG accounting for only a modest difference by race in this variable. Interestingly, the incidence of the Metabolic Syndrome [35] and of coronary heart disease [36] is only slightly greater in AA than CA women despite AA women having much greater rates of overweight/obesity. This, however, may be more closely related to differences in other risk factors such as high blood pressure and diabetes which tend to be higher in AA women.

Tchernof and Després [3] have maintained that VAT plays an important role in moderating the relationship between sex steroids and coronary risk factors. However, when we controlled for VAT, all significant correlations with $T T$ remained. They also remained when we controlled for insulin. Thus, the mechanisms responsible for the significant relationship found in AA women require further study. Past research shows no consensus opinion regarding the relationship between endogenous steroids and serum lipids or lipoproteins [3]. Our finding of a significant relationship in AA women adds to the controversy surrounding this issue.

As expected, significant and positive relationships were observed between FAI and insulin/glucose status, as well as central obesity measures (SAT and waist) $[1,2,10,11]$. In AA women, a different case scenario was observed. Similar to that shown with TT, FAI was significantly related to DBP and atherogenic lipoproteins (total cholesterol, LDL, and Apo B) but not measures of glucose/insulin status.
Upon further evaluation, CA compared to AA women showed roughly double the magnitude of correlation between FAI and either fasting insulin or HOMA IR. Furthermore, glucose shared almost $20 \%$ of its variance with FAI in CA women, while in AA women, there was virtually no shared variance between glucose and FAI. This discrepancy accounted for an $\mathrm{FAI} /$ glucose relationship ten times greater in CA than AA women.

Other studies [37, 38] have suggested that AA women are more insulin resistant than CA women even after adjustment for VAT [37]. In postmenopausal women, Ryan and colleagues [37] have shown a $60 \%$ lower glucose uptake concomitant with a higher insulin level in AA compared to CA women. Although there were no significant differences between fasting insulin or HOMA IR in CA and AA women, our data reaffirms racial differences in the relationship between FAI and the MRP specifically with regard to the FAI/glucose relationship. This is particularly relevant since diabetes risk is 2.4 times greater in AA than CA women [7]. Further research is warranted to support the mechanisms responsible for these differences.

Our work contrasted with the findings of Pedersen et al. [2] who did not find significant relationships between FAI and the MRP, independent of VAT. The only exception was the relationship between HDL and FAI. In fact, our results showed that insulin played a more critical role than 
TABle 3: Pearson correlation coefficients between free androgen index (log transformed) and the metabolic risk profile after adjusting for age.

\begin{tabular}{|c|c|c|c|c|}
\hline Metabolic risk profile & $\begin{array}{c}\text { Caucasian American } \\
\text { women ( } r \text { value })\end{array}$ & $P$ value & $\begin{array}{c}\text { African American } \\
\text { women ( } r \text { value })\end{array}$ & $P$ value \\
\hline BMI $\left(\mathrm{kg} / \mathrm{m}^{2}\right)$ & 0.188 & .279 & 0.149 & .440 \\
\hline WAIST (cm) & 0.337 & .048 & 0.323 & .087 \\
\hline SAT (L) & 0.376 & .026 & -0.080 & .682 \\
\hline $\operatorname{VAT}(\mathrm{L})$ & 0.308 & .072 & 0.316 & .095 \\
\hline Glucose $(\mathrm{mmol} / \mathrm{L})$ & 0.519 & .002 & -0.044 & .829 \\
\hline Insulin $(\mathrm{u} \mu / \mathrm{mL})$ & 0.436 & .0100 & 0.264 & .192 \\
\hline HOMA IR (ratio) & 0.478 & .004 & 0.236 & .247 \\
\hline $\mathrm{SBP}(\mathrm{mmHg})$ & -0.197 & .257 & -0.301 & .112 \\
\hline $\mathrm{DBP}(\mathrm{mmHg})$ & -0.211 & .223 & -0.435 & .018 \\
\hline $\mathrm{TG}(\mathrm{mg} / \mathrm{dL})$ & 0.195 & .269 & -0.067 & .746 \\
\hline $\begin{array}{l}\text { Total cholesterol } \\
(\mathrm{mmol} / \mathrm{L})\end{array}$ & -0.196 & .269 & -0.504 & .009 \\
\hline $\mathrm{HDL}(\mathrm{mmol} / \mathrm{L})$ & -0.349 & .043 & -0.249 & .220 \\
\hline $\mathrm{HDL}_{2}(\mathrm{mmol} / \mathrm{L})$ & -0.211 & .231 & -0.238 & .243 \\
\hline $\mathrm{LDL}(\mathrm{mmol} / \mathrm{L})$ & -0.253 & .149 & -0.499 & .010 \\
\hline LDL pps (nmol/L) & -0.092 & .598 & -0.028 & .891 \\
\hline Apo B (mg/dL) & -0.151 & .192 & -0.428 & .029 \\
\hline $\begin{array}{l}\text { Total } \\
\text { cholesterol/HDL-C }\end{array}$ & 0.035 & .844 & -0.297 & .141 \\
\hline $\mathrm{Lp}(\mathrm{a})$ & -0.097 & .594 & -0.125 & .542 \\
\hline
\end{tabular}

BMI, body mass index, WAIST, waist circumference, SAT, subcutaneous adipose tissue volume, VAT, visceral adipose tissue volume, SBP systolic blood pressure, DBP, diastolic blood pressure, HOMA IR, insulin resistance calculated as (insulin $\times$ glucose) $\div 22.5$; HDL, high-density lipoprotein cholesterol, $\mathrm{HDL}_{2}$, high density lipoprotein subfraction 2, LDL, low density lipoprotein cholesterol, LDL pps, low-density lipoprotein peak particle size, Apo B, apolipoprotein B, TG, triglycerides, log transformed.

VAT in modulating these relationships. Upon controlling for insulin, the relationship between FAI and all lipid variables in both CA and AA women was negated. These findings are supported by Haffner and colleagues [11,39] who proposed that insulin plays a more primary role in relation to the sex steroid milieu. Insulin is reported to stimulate androgen production in the ovary, and bioavailable androgens may be more closely associated with insulin and insulin resistance $[11,40]$. Androgens also play a role in modulating hepatic lipase activity [2] which would explain subsequent changes in the relationship between FAI and HDL, as well as TG after controlling for insulin. It does not explain how insulin modulates the relationship between FAI and other lipids such as total cholesterol, LDL, or APO B, shown in AA women.

In CA women, the same four variables related to FAI were also related to SHBG (waist, glucose, insulin, and HOMA IR). Relationships were additionally observed between SHBG and VAT. These relationships confirm the deleterious effects of low SHBG/high FAI reported in many previous studies [2, $4,8,10]$. Furthermore, insulin has been shown to suppress hepatocellular SHBG production [41] and both insulin and VAT have been reported to impact the relationship between SHBG and the MRP $[1,2,10]$. Controlling for insulin, but not VAT, negated all significant relationships between SHBG and the MRP in CA women. This indicates the importance of insulin in modulating relationships between SHBG and variables associated with CVD risk.
In AA women, the only significant relationship observed with SHBG was waist circumference. At first glance our findings mirror the results of those by Berman et al. [9] who found significant relationships between SHBG and the MRP in overweight/obese postmenopausal CA but not AA women. They surmised that differences were related to a higher postheparin lipase activity and a lower level of hepatic lipase in AA women which would explain a greater capacity to clear TG's from circulation in their AA women. Our study showed a lower VAT which was related to lower TG in AA women. Thus, the mechanism proposed for racial differences in their postmenstrual women may not be applicable to premenopausal women in our study.

Upon further examination, however, there was a trend toward significance between SHBG and insulin as well as HOMA IR, HDL, and VAT in AA women (see Table 4). Although these correlations were somewhat lower in AA than CA women, they do not negate the fact that some clinical associations may be occurring in AA women. In the SWAN study [8] and the study by Sherif and colleagues [34], they did find significant relationships between SHBG and HDL in AA women, which were both lower than that of AA women in the present study. This is likely due to the much larger number of subjects in the SWAN study and slightly higher number of AA subjects in the study by Sherif [34]. Subjects in the SWAN study [8] also showed considerably lower BMI levels than our study. Certainly, a 
TABle 4: Pearson correlation coefficients between sex hormone binding globulin (log transformed) and the metabolic risk profile after adjusting for age.

\begin{tabular}{|c|c|c|c|c|}
\hline Metabolic risk profile & $\begin{array}{c}\text { Caucasian American } \\
\text { women ( } r \text { value })\end{array}$ & $P$ value & $\begin{array}{c}\text { African American } \\
\text { women ( } r \text { value })\end{array}$ & $P$ value \\
\hline BMI $\left(\mathrm{kg} / \mathrm{m}^{2}\right)$ & -0.267 & .121 & -0.171 & .375 \\
\hline WAIST (cm) & -0.365 & .031 & -0.424 & .022 \\
\hline SAT (L) & -0.230 & .184 & 0.045 & .817 \\
\hline $\operatorname{VAT}(\mathrm{L})$ & -0.478 & .004 & -0.315 & .096 \\
\hline Glucose $(\mathrm{mmol} / \mathrm{L})$ & -0.442 & .009 & -0.029 & .89 \\
\hline Insulin $(\mathrm{u} \mu / \mathrm{mL})$ & -0.527 & .001 & -0.379 & .056 \\
\hline HOMA IR (ratio) & -0.542 & .001 & -0.339 & .090 \\
\hline $\mathrm{SBP}(\mathrm{mmHg})$ & -0.191 & .271 & 0.183 & .341 \\
\hline $\mathrm{DBP}(\mathrm{mmHg})$ & -0.183 & .800 & 0.257 & .179 \\
\hline $\mathrm{TG}(\mathrm{mg} / \mathrm{dL})$ & -0.331 & .056 & -0.137 & .504 \\
\hline $\begin{array}{l}\text { Total cholesterol } \\
(\mathrm{mmol} / \mathrm{L})\end{array}$ & -0.040 & .820 & -0.192 & .373 \\
\hline HDL (mmol/L) & 0.262 & .135 & 0.335 & .094 \\
\hline $\mathrm{HDL}_{2}(\mathrm{mmol} / \mathrm{L})$ & 0.300 & .085 & 0.100 & .627 \\
\hline $\mathrm{LDL}(\mathrm{mmol} / \mathrm{L})$ & 0.117 & .511 & 0.182 & .373 \\
\hline LDL pps (nmol/L) & 0.164 & .355 & -0.233 & .252 \\
\hline Apo B (mg/dL) & -0.013 & .943 & -0.079 & .700 \\
\hline Total cholesterol/HDL & -0.111 & .533 & -0.021 & .917 \\
\hline $\operatorname{Lp}(\mathrm{a})$ & 0.071 & .696 & -0.202 & .322 \\
\hline
\end{tabular}

BMI, body mass index, WAIST, waist circumference, SAT, subcutaneous adipose tissue volume, VAT, visceral adipose tissue volume, SBP systolic blood pressure, DBP, diastolic blood pressure, HOMA IR, insulin resistance calculated as (insulin $\times$ glucose) $\div 22.5$; HDL, high-density lipoprotein cholesterol, $\mathrm{HDL}_{2}$, high-density lipoprotein subfraction 2, LDL, low-density lipoprotein cholesterol, LDL pps, low-density lipoprotein peak particle size, Apo B, apolipoprotein B, TG, triglycerides, log transformed.

higher number of AA women in our study would have been helpful.

It remains unclear as to why some of the aforementioned relationships, particularly with regard to glucose/insulin status, were considerably stronger in CA than AA women. Investigators in the SWAN study [8] concluded that relationships were, in general, similar across racial lines being stronger in certain racial groups and weaker in others. With regard to the relationship between SHBG and the MRP, our results tended to reinforce their findings.

There were, however, several limitations noted with respect to this study. First, all relationships were based upon a single blood draw at one time point. It would have been better to obtain multiple samples particularly in a crosssectional study. Furthermore, an $n$ of 66 subjects which included a biracial group of women, limited our power. Since this was a cross sectional study, it was difficult to determine any temporal or causal relationships between sex steroids, SHBG, and the MRP. We could only speculate primary relationships based upon the strength of the correlation analysis before and after controlling for potential confounders, that is, VAT and insulin. Since many variables were correlated with each other, it was difficult to determine if some relationships were operating independently or via their association with other metabolic variables. Although women were recruited from the same medical institution and consisted of nurses, nurse assistants, and technicians, it would have been helpful to examine the socioeconomic status of $\mathrm{CA}$ and $\mathrm{AA}$ women. A few women in both groups were in their mid to late 40's and may have been perimenopausal. This transition period prior to menopause may be linked to a progressive reduction in ovarian function, reduced estrogen levels, and stable androgen levels. Estrogen measurements would have been helpful. Although age was controlled for in all analysis, this may have affected the androgen/estrogen balance in several women. Furthermore, blood sampling was done randomly and not at uniform times in the menstrual cycle. Since total and LDL cholesterol show variability across follicular and luteal phases of the cycle [41], it may have affected relationships or lack thereof between the sex steroid milieu and serum lipoproteins in $\mathrm{CA}$ and $\mathrm{AA}$ women. It would have been preferable to sample all women at the same time in their menstrual cycle; however, it is hoped that the random selection of testing dates and blood withdrawal will have prevented any undue influences in our results. Finally, free testosterone was not measured directly but computed indirectly as FAI using both TT and SHBG in the formulation. Although FAI is considered a viable correlate of free testosterone, the direct measurement of free testosterone would have been preferable, particularly in women, in the low normal range. Although investigators sampled first thing in the morning following an overnight fast to reduce variability in androgen levels, it is always preferable to measure free testosterone 
directly. Fortunately other confounding variables such as physical activity, smoking, alcohol, and stress known to affect the MRP were not found to be significantly different between groups.

\section{Conclusions}

Given aforementioned limitations, several important findings were noted. First, independent of VAT or insulin, TT showed several significant and cardioprotective relationships with several variables in the MRP, in both CA and AA women. Second, our study reinforced racial differences in the relationship between androgens and the MRP. This was evidenced by the significant relationships found between TT and FAI and serum lipoproteins in AA women that were absent in CA women. There were also significant relationships found between FAI and glucose/insulin status in CA women that were absent in AA women. Furthermore, the aforementioned relationships were not mediated by VAT but were more strongly influenced by insulin. Racial differences were also noted with respect to the strength of the relationship observed between SHBG and the MRP. These tended to be stronger in CA women. Future research is recommended to further elucidate the mechanisms responsible for these contrasting relationships and trends in premenopausal CA and AA women.

\section{Disclosure}

None of the authors reported any disclosures and there is no conflict of interest that would prejudice the impartiality of the research reported.

\section{Acknowledgments}

The authors wish to acknowledge the assistance of Dr. Steven Deprima and his staff at HealthSouth Doctors' Hospital for their help in providing the magnetic resonance imaging information on all study participants.

\section{References}

[1] A. Tchernof, M. J. Toth, and E. T. Poehlman, "Sex hormonebinding globulin levels in middle-aged premenopausal women: associations with visceral obesity and metabolic profile," Diabetes Care, vol. 22, no. 11, pp. 1875-1881, 1999.

[2] S. B. Pedersen, J. D. Borglum, K. Brixen, and B. Richelsen, "Relationship between sex hormones, body composition and metabolic risk parameters in premenopausal women," European Journal of Endocrinology, vol. 133, no. 2, pp. 200206, 1995.

[3] A. Tchernof and J.-P. Després, "Sex steroid hormones, sex hormone-binding globulin, and obesity in men and women," Hormone and Metabolic Research, vol. 32, no. 11-12, pp. 526536, 2000

[4] R. J. Bell, S. L. Davison, M.-A. Papalia, D. P. McKenzie, and S. R. Davis, "Endogenous androgen levels and cardiovascular risk profile in women across the adult life span," Menopause, vol. 14, no. 4, pp. 630-638, 2007.
[5] J. Sundquist, M. A. Winkleby, and S. Pudaric, "Cardiovascular Disease Risk Factors Among Older Black, Mexican-American, and White Women and Men: an analysis of NHANES III, 1988-1994," Journal of the American Geriatrics Society, vol. 49, no. 2, pp. 109-116, 2001.

[6] C. L. Ogden, M. D. Carroll, and K. M. Flegal, "Epidemiologic trends in overweight and obesity," Endocrinology and Metabolism Clinics of North America, vol. 32, no. 4, pp. 741760, 2003.

[7] F. L. Brancati, W. H. L. Kao, A. R. Folsom, R. L. Watson, and M. Szklo, "Incident type 2 diabetes mellitus in African American and white adults: the atherosclerosis risk in communities study," Journal of the American Medical Association, vol. 283, no. 17, pp. 2253-2259, 2000.

[8] K. Sutton-Tyrrell, R. P. Wildman, K. A. Matthews et al., "Sex hormone-binding globulin and the free androgen index are related to cardiovascular risk factors in multiethnic premenopausal and perimenopausal women enrolled in the study of women across the nation (SWAN)," Circulation, vol. 111, no. 10, pp. 1242-1249, 2005.

[9] D. M. Berman, L. M. Rodrigues, B. J. Nicklas, A. S. Ryan, K. E. Dennis, and A. P. Goldberg, "Racial disparities in metabolism, central obesity, and sex hormone-binding globulin in postmenopausal women," Journal of Clinical Endocrinology and Metabolism, vol. 86, no. 1, pp. 97-103, 2001.

[10] S. M. Haffner, M. S. Katz, M. P. Stern, and J. F. Dunn, "Association of decreased sex hormone binding globulin and cardiovascular risk factors," Arteriosclerosis, vol. 9, no. 1, pp. 136-143, 1989.

[11] S. M. Haffner, "Sex hormone-binding protein, hyperinsulinemia, insulin resistance and noninsulin-dependent diabetes," Hormone Research, vol. 45, no. 3-5, pp. 233-237, 1996.

[12] World Health Organization, "Physical Status: The Use and Interpretation of Anthropometry," Report of a WHO Expert Committee, World Health Organization, Geneva, Switzerland, 1995.

[13] Manual of Laboratory Operations Lipid Research Clinic Program: Lipid and Lipoprotein Analysis, DHEW, Washington, DC, USA, 1974.

[14] G. R. Warnick and J. J. Albers, "A comprehensive evaluation of the heparin-manganese precipitation procedure for estimating high density lipoprotein cholesterol," Journal of Lipid Research, vol. 19, no. 1, pp. 65-76, 1978.

[15] L. I. Gidez, G. J. Miller, and M. Burstein, "Separation and quantitation of subclasses of human plasma high density lipoproteins by a simple precipitation procedure," Journal of Lipid Research, vol. 23, no. 8, pp. 1206-1223, 1982.

[16] M. W. McGowan, J. D. Artiss, D. R. Strandbergh, and B. Zak, "A peroxidase-coupled method for the colorimetric determination of serum triglycerides," Clinical Chemistry, vol. 29, no. 3, pp. 538-542, 1983.

[17] W. T. Friedewald, R. I. Levy, and D. S. Fredrickson, "Estimation of the concentration of low-density lipoprotein cholesterol in plasma, without use of the preparative ultracentrifuge," Clinical Chemistry, vol. 18, no. 6, pp. 499-502, 1972.

[18] R. M. Krauss and D. J. Burke, "Identification of multiple subclasses of plasma low density lipoproteins in normal humans," Journal of Lipid Research, vol. 23, no. 1, pp. 97-104, 1982.

[19] S. M. Haffner, H. Miettinen, and M. P. Stern, "The homeostasis model in the San Antonio Heart Study," Diabetes Care, vol. 20, no. 7, pp. 1087-1092, 1997. 
[20] L. F. Blight, S. J. Judd, and G. H. White, "Relative diagnostic value of serum non-SHBG-bound testosterone, free androgen index and free testosterone in the assessment of mild to moderate hirsutism," Annals of Clinical Biochemistry, vol. 26, no. 4, pp. 311-316, 1989.

[21] A. Vermeulen, L. Verdonck, and J. M. Kaufman, "A critical evaluation of simple methods for the estimation of free testosterone in serum," Journal of Clinical Endocrinology and Metabolism, vol. 84, no. 10, pp. 3666-3672, 1999.

[22] R. S. Paffenbarger Jr., S. N. Blair, I.-M. Lee, and R. T. Hyde, "Measurement of physical activity to assess health effects in free-living populations," Medicine and Science in Sports and Exercise, vol. 25, no. 1, pp. 60-70, 1993.

[23] R. Rosmond, L. Lapidus, and P. Björntorp, "The influence of occupational and social factors on obesity and body fat distribution in middle-aged men," International Journal of Obesity, vol. 20, no. 7, pp. 599-607, 1996.

[24] P. J. Brantley, L. S. Dietz, G. T. McKnight, G. N. Jones, and R. Tulley, "Convergence between the daily stress inventory and endocrine measures of stress," Journal of Consulting and Clinical Psychology, vol. 56, no. 4, pp. 549-551, 1988.

[25] SPSS for Windows, release 10.1, SPSS, Inc, Chicago, Ill, USA, 2000.

[26] J. M. Conway, S. Z. Yanovski, N. A. Avila, and V. S. Hubbard, "Visceral adipose tissue differences in black and white women," American Journal of Clinical Nutrition, vol. 61, no. 4, pp. 765-771, 1995.

[27] J. B. Albu, L. Murphy, D. H. Frager, J. A. Johnson, and F. X. PiSunyer, "Visceral fat and race-dependent health risks in obese nondiabetic premenopausal women," Diabetes, vol. 46, no. 3 , pp. 456-462, 1997.

[28] H. J. Dowling and F. X. Pi-Sunyer, "Race-dependent health risks of upper body obesity," Diabetes, vol. 42 , no. 4 , pp. 537 543, 1993.

[29] D. Goodman-Gruen and E. Barrett-Connor, "Total but not bioavailable testosterone is a predictor of central adiposity in postmenopausal women," International Journal of Obesity, vol. 19, no. 5, pp. 293-298, 1995.

[30] P. M. Sarrel, "Cardiovascular aspects of androgens in women," Seminars in Reproductive Endocrinology, vol. 16, no. 2, pp. 121128, 1998.

[31] T. Kienitz and M. Quinkler, "Testosterone and blood pressure regulation," Kidney and Blood Pressure Research, vol. 31, no. 2, pp. 71-79, 2008.

[32] C. Livingstone and M. Collison, "Sex steroids and insulin resistance," Clinical Science, vol. 102, no. 2, pp. 151-166, 2002.

[33] M. J. Stampfer, F. M. Sacks, S. Salvini, W. C. Willett, and C. H. Hennekens, "A prospective study of cholesterol, apolipoproteins, and the risk of myocardial infarction," New England Journal of Medicine, vol. 325, no. 6, pp. 373-381, 1991.

[34] K. Sherif, H. Kushner, and B. E. Falkner, "Sex hormonebinding globulin and insulin resistance in African-American women," Metabolism, vol. 47, no. 1, pp. 70-74, 1998.

[35] E. S. Ford, W. H. Giles, and W. H. Dietz, "Prevalence of the metabolic syndrome among US adults: findings from the third National Health and Nutrition Examination Survey," Journal of the American Medical Association, vol. 287, no. 3, pp. 356359, 2002.

[36] D. Lloyd-Jones, R. J. Adams, T. M. Brown et al., "Heart disease and stroke statistics-2010 update: a report from the American Heart Association," Circulation, vol. 121, no. 7, pp. 948-954, 2010.
[37] A. S. Ryan, B. J. Nicklas, and D. M. Berman, "Racial differences in insulin resistance and mid-thigh fat deposition in postmenopausal women," Obesity Research, vol. 10, no. 5, pp. 336-344, 2002.

[38] J. C. Lovejoy, J. A. de la Bretonne, M. Klemperer, and R. Tulley, "Abdominal fat distribution and metabolic risk factors: effects of race," Metabolism, vol. 45, no. 9, pp. 1119-1124, 1996.

[39] S. M. Haffner, "Sex hormones, obesity, fat distribution, type 2 diabetes and insulin resistance: epidemiological and clinical correlation," International Journal of Obesity, vol. 24, no. 2, pp. S56-S58, 2000.

[40] R. L. Barbieri and K. J. Ryan, "Hyperandrogenism, Insulin Resistance, and Acanthosis Nigricans Syndrome. A Common Endocrinopathy with Distinct Pathophysiologic Features," American Journal of Obstetrics and Gynecology, vol. 147, no. 1, pp. 90-101, 1983.

[41] S. R. Plymate, L. A. Matej, R. E. Jones, and K. E. Friedl, "Inhibition of sex hormone-binding globulin production in the human hepatoma (Hep G2) cell line by insulin and prolactin," Journal of Clinical Endocrinology and Metabolism, vol. 67 , no. 3 , pp. 460-464, 1988. 


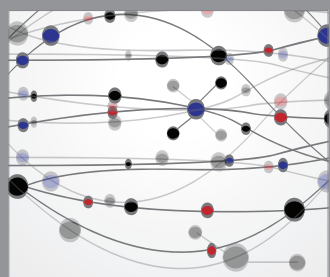

The Scientific World Journal
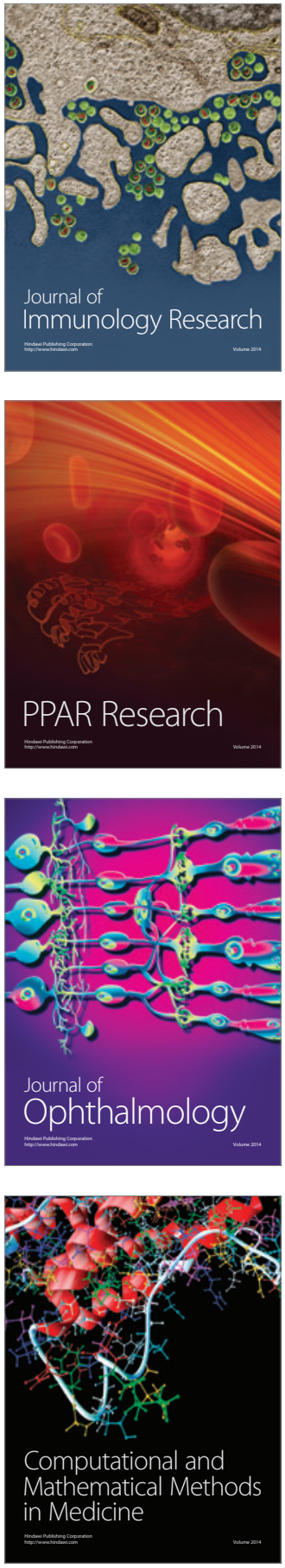

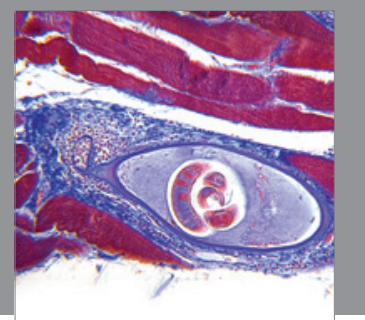

Gastroenterology

Research and Practice
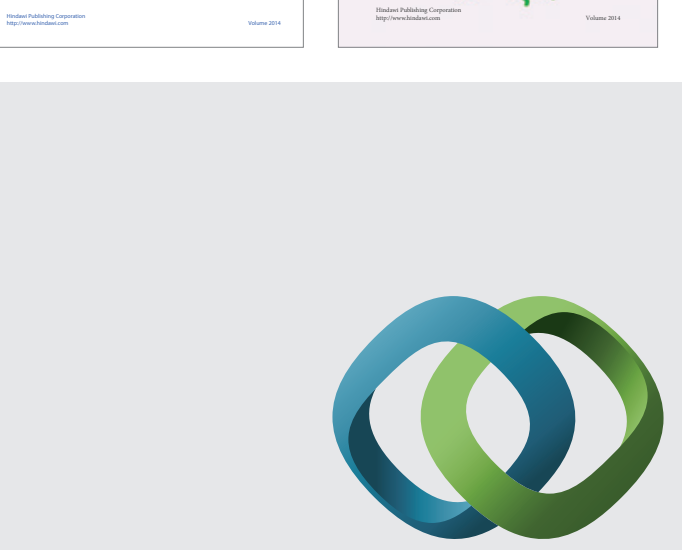

\section{Hindawi}

Submit your manuscripts at

http://www.hindawi.com
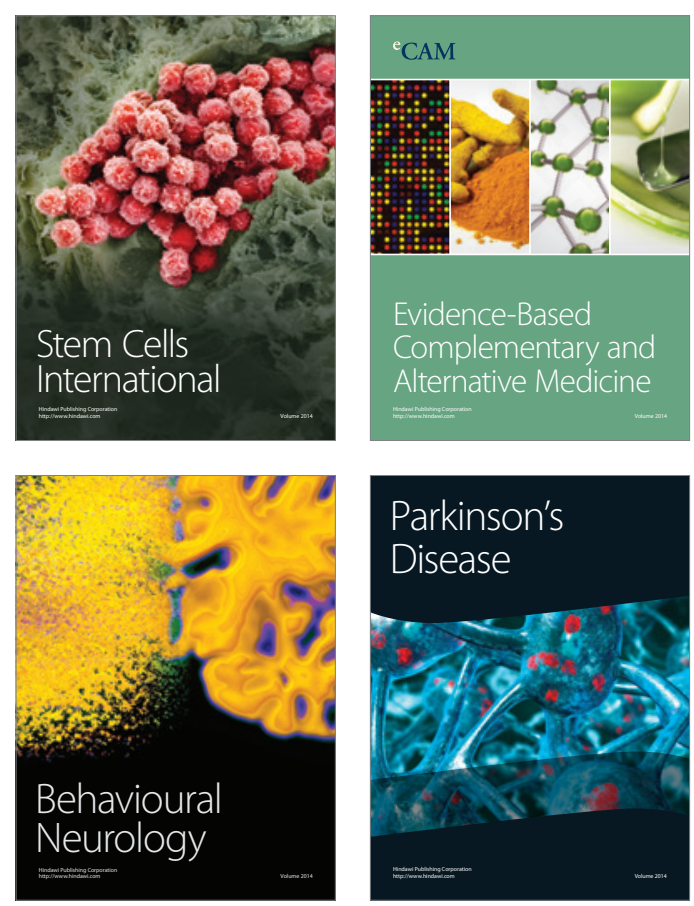

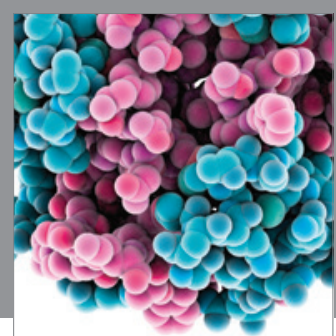

Journal of
Diabetes Research

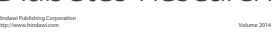

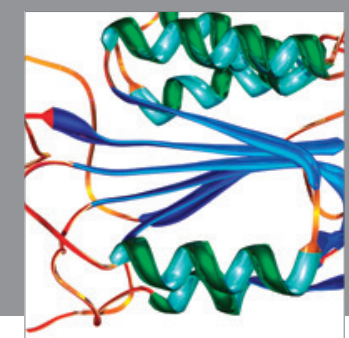

Disease Markers
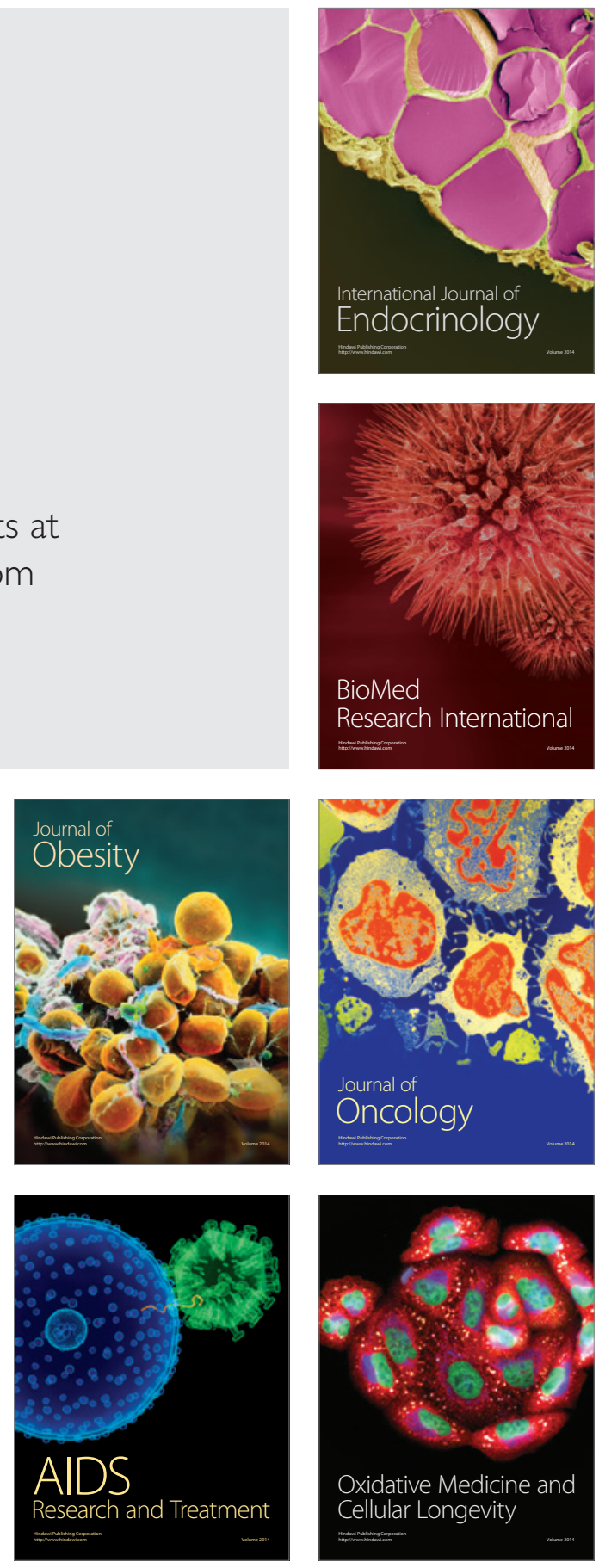